\title{
The odd Fréchet inverse Rayleigh distribution: statistical properties and applications
}

\author{
M. Elgarhy ${ }^{a, *}$, Sharifah Alrajhib \\ ${ }^{a}$ Vice Presidency for Graduate Studies and Scientific Research, University of Jeddah, Jeddah, KSA. \\ ${ }^{b}$ Statistics Department, Faculty of Science, King AbdulAziz University, Jeddah, KSA.
}

\begin{abstract}
We propose a new distribution with two parameters called the odd Fréchet inverse Rayleigh (OFIR) distribution. The new model can be more flexible. Several of its statistical properties are studied. The maximum likelihood (ML) estimation is used to drive estimators of OFIR parameters. The importance and flexibility of the new model is assessed using one real data set.
\end{abstract}

Keywords: Odd Fréchet family, inverse Rayleigh distribution, moments, maximum likelihood.

2010 MSC: 60E05, 62E10, 62N05.

(C)2019 All rights reserved.

\section{Introduction}

An appropriate comprehensive lifetime model is often of concentration in the analysis of data. Trayer [19] introduced a distribution in order to model reliability and survival data sets, named inverse Rayleigh distribution. After that, inverse Rayleigh (IR) distribution was championed by Voda [20]. He discussed its properties and ML estimator of the scale parameter. Further, Gharraph [8] provided closed-form expressions for the mean, harmonic mean, geometric mean, mode and the median of this distribution.

Lots of works have been studied in the literature on IR distribution. Gharraph [8] and Hassan et al. [13] estimated the parameters using classical and Bayesian estimation methods.

Beta inverse Rayleigh distribution was studied by Leao et al. [16], Ahmed et al. [4] introduced a generalization of the inverse Rayleigh distribution, modified inverse Rayleigh distribution studied by Khan [14], Khan and King [15] studied transmuted modified inverse Rayleigh distribution, Haq [11] introduced transmuted exponentiated inverse Rayleigh distribution, and Kumaraswamy exponentiated inverse Rayleigh distribution was studied by Haq [10].

The probability density function (pdf) and cumulative distribution function (cdf) of IR distribution are given by

$$
g(x: \alpha)=\frac{2 \alpha}{x^{3}} e^{-\frac{\alpha}{x^{2}}}, \quad x, \alpha>0,
$$

\footnotetext{
${ }^{*}$ Corresponding author

Email addresses: m_elgarhy85@yahoo.com (M. Elgarhy), saalrajhi@kau.edu.sa (Sharifah Alrajhi)

doi: $10.22436 /$ jnsa.012.05.03
}

Received: 2018-10-03 Revised: 2018-10-30 Accepted: 2018-11-16 
and

$$
G(x: \alpha)=e^{-\frac{\alpha}{x^{2}}}, \quad x, \alpha>0 .
$$

Recently, the odd Fréchet generated family of distributions (OF-G) has been proposed by Haq and Elgarhy [12] in order to get more flexibility to a family of distributions. The cdf of a continuous random variable $\mathrm{X}$ having OF-G is given by:

$$
F(x: \theta, \xi)=\int_{0}^{\left[\frac{G(x ; \xi)}{1-G(x ; \xi)}\right]} \frac{\theta}{x^{\theta+1}} e^{-x^{-\theta}} d x=e^{-\left[\frac{1-G(x ; \xi)}{G(x ; \xi)}\right]^{\theta}}, \quad x \in R, \theta>0 .
$$

The corresponding pdf to (1.2) is given by

$$
f(x: \theta, \xi)=\frac{\theta g(x ; \xi)[1-G(x ; \xi)]^{\theta-1}}{G(x ; \xi)^{\theta+1}} e^{-\left[\frac{1-G(x ; \xi)}{G(x ; \xi)}\right]^{\theta}},
$$

where $g(x: \xi)$ considers a pdf of baseline distribution. Hereafter, a random variable $X$ with density function (1.3) is denoted by $X \sim O F-G(\theta, \xi)$.

The hazard rate function (hrf) of the OF-G family is

$$
h(x: \theta, \xi)=\frac{\theta g(x ; \xi)[1-G(x ; \xi)]^{\theta-1} e^{-\left[\frac{1-G(x ; \xi)}{G(x ; \xi)}\right]^{\theta}}}{G(x ; \xi)^{\theta+1}\left[1-e^{-\left[\frac{1-G(x ; \xi)}{G(x ; \xi)}\right]^{\theta}}\right]} .
$$

In this paper, we define a new lifetime model called the OFIR distribution. We hope that it will attract wider applications in engineering, medicine, and other areas of research. This paper is organized as follows. In Sections 2 and 3, we study the OFIR and calculate its properties. The ML method is applied to drive the estimators of the model parameters in Section 4. Numerical results are carried out obtain the estimates of the model parameters of OFIR distribution in Section 5. The analyses of one real data set is employed in Section 6. Concluding remarks appear in Section 7.

\section{The new model}

The cdf of OFIR distribution with set of parameters $\varphi=(\alpha, \theta)$ is obtained by substituting (1.1) in (1.2) as follows

$$
F(x ; \theta, \alpha)=e^{-\left[e^{\frac{\alpha}{x^{2}}}-1\right]^{\theta}}, \quad x, \alpha, \theta>0
$$

The corresponding pdf to (2.1) is given by

$$
f(x ; \theta, \alpha)=\frac{2 \theta \alpha}{x^{3}} e^{\frac{\alpha}{x^{2}}}\left[e^{\frac{\alpha}{x^{2}}}-1\right]^{\theta-1} e^{-\left[e^{\frac{\alpha}{x^{2}}}-1\right]^{\theta}}, \quad x, \alpha, \theta>0 .
$$

Also, the survival function (sf), hrf, reversed hrf, and cumulative hrf of $\mathrm{X}$ are given, respectively, as follows:

$$
\begin{aligned}
& R(x ; \theta, \alpha)=1-e^{-\left[e^{\frac{\alpha}{x^{2}}}-1\right]^{\theta}}, \\
& h(x ; \theta, \alpha)=\frac{\frac{2 \theta \alpha}{x^{3}} e^{\frac{\alpha}{x^{2}}}\left[e^{\frac{\alpha}{x^{2}}}-1\right]^{\theta-1} e^{-\left[e^{\frac{\alpha}{x^{2}}}-1\right]^{\theta}}}{1-e^{-\left[e^{\frac{\alpha}{x^{2}}}-1\right]^{\theta}}},
\end{aligned}
$$




$$
\tau(x ; \theta, \alpha)=\frac{2 \theta \alpha}{\chi^{3}} e^{\frac{\alpha}{x^{2}}}\left[e^{\frac{\alpha}{x^{2}}}-1\right]^{\theta-1}
$$

and

$$
H(x ; \theta, \alpha)=-\ln \left(1-e^{-\left[e^{\frac{\alpha}{x^{2}}}-1\right]^{\theta}}\right)
$$

Hereafter, a random variable $X$ that follows the distribution in (2.2) is denoted by $X \sim$ OFIR $(\varphi)$, where $\varphi=(\theta, \alpha)$. Some descriptive pdf and hrf plots of $X \sim \operatorname{OFIR}(\varphi)$ are illustrated below for specific parameter choices of $\varphi$ (see Figure 1).
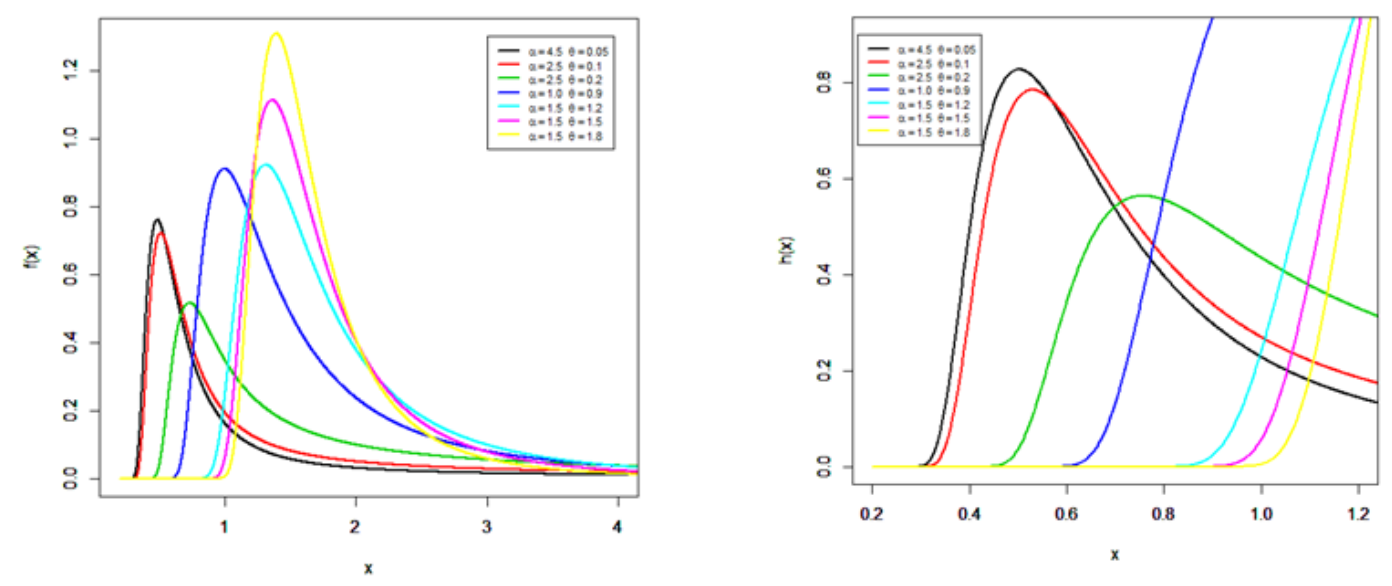

Figure 1: Plots of the pdf and hrf of the (OFIR) distribution for different values of parameters.

From Figure 1, we conclude that pdf of OFIR distribution can be upside-down and right skewed. Also, the hrf of OFIR distribution can be J-shaped and unimodal as seen from Figure 1.

\section{Statistical properties}

In this section some properties of the OFIR distribution are obtained.

\subsection{Quantile and median}

The quantile function, say $\mathrm{Q}(\mathrm{u})=\mathrm{F}^{-1}(\mathrm{u})$ of $\mathrm{X}$ is given by

$$
u=e^{-\left[e^{\frac{\alpha}{(Q(u))^{2}}}-1\right]^{\Theta}}
$$

after some simplifications, it reduces to the following form

$$
Q(u)=\sqrt{\frac{\alpha}{\ln \left(1+\left[\ln \left(\frac{1}{u}\right)\right]^{\frac{1}{\theta}}\right)}},
$$

where, $u$ is considered as a uniform random variable on the unit interval $(0,1)$. by

In particular, the median can be derived from (3.1) by setting $u=0.5$. That is, the median $(\mathrm{M})$ is given

$$
M=\sqrt{\frac{\alpha}{\ln \left(1+[\ln (2)]^{\frac{1}{\theta}}\right)}} .
$$




\subsection{Linear representation}

In this subsection representations of the pdf and cdf for OFIR distribution are derived.

Haq and Elgarhy [12] expressed the equation (1.3) as

$$
f(x)=\sum_{k=0}^{\infty} \eta_{k} g(x, \xi) G(x, \xi)^{k},
$$

where

$$
\eta_{k}=\sum_{i, j=0}^{\infty} \frac{\theta(-1)^{i+k}}{i !}\left(\begin{array}{c}
\theta(i+1)+j \\
j
\end{array}\right)\left(\begin{array}{c}
\theta(i+1)+j-1 \\
k
\end{array}\right) .
$$

By inserting equation (2.2) in equation (3.2) we can rewite the OFIR as a linear combination of IR distribution as

$$
f(x)=\sum_{k=0}^{\infty} \frac{w_{k}}{x^{3}} e^{-\frac{\alpha(k+1)}{x^{2}}}
$$

where $w_{k}=2 \alpha \eta_{k}$.

\subsection{Moments}

If $\mathrm{X}$ has the pdf (3.3), then its rthmoment can be calculated through the following relation

$$
\mu_{r}^{\prime}=E\left(X^{r}\right)=\int_{-\infty}^{\infty} x^{r} f(x ; \varphi) d x
$$

Substituting (3.3) into (3.4) yields:

$$
\mu_{r}^{\prime}=E\left(X^{r}\right)=\sum_{k=0}^{\infty} w_{k} \int_{0}^{\infty} x^{r-3} e^{-\alpha(k+1) x^{-2}} d x
$$

Let $y=x^{-2}$, then

$$
\mu_{r}^{\prime}=\sum_{k=0}^{\infty} \frac{w_{k}}{2} \int_{0}^{\infty} y^{\frac{-r}{2}} e^{-\alpha(k+1) y} d y
$$

then $\mu_{r}^{\prime}$ becomes

$$
\mu_{r}^{\prime}=\sum_{k=0}^{\infty} \frac{w_{k} \Gamma\left(1-\frac{r}{2}\right)}{2[\alpha(k+1)]^{1-\frac{r}{2}}}, \quad r<2 .
$$

The moment generating function of OFIR distribution is obtained through the following relation

$$
M_{X}(t)=\sum_{r=0}^{\infty} \frac{t^{r}}{r !} E\left(X^{r}\right)=\sum_{r, k=0}^{\infty} \frac{t^{r}}{r !} \frac{w_{k} \Gamma\left(1-\frac{r}{2}\right)}{2[\alpha(k+1)]^{1-\frac{r}{2}}}, r<2
$$

\subsection{Incomplete and conditional moments}

The main application of the first incomplete moment refers to the Bonferroni and Lorenz curves. These curves are very useful in economics, reliability, demography, insurance and medicine. The answers to many important questions in economics require more than just knowing the mean of the distribution, but its shape as well. This is obvious not only in the study of econometrics but in other areas as well. The incomplete moments, say $\varpi_{s}(t)$, is given by

$$
\varpi_{s}(t)=\int_{0}^{t} x^{s} f(x ; \varphi) d x
$$


Using (3.3), then $\phi_{s}(t)$ can written as follows

$$
\varpi_{s}(t)=\sum_{k=0}^{\infty} w_{k} \int_{0}^{t} x^{s-3} e^{-\alpha(k+1) x^{-2}} d x .
$$

Then, using the lower incomplete gamma function, we obtain

$$
\varpi_{s}(t)=\sum_{k=0}^{\infty} w_{k} \frac{v\left(1-\frac{s}{2}, \alpha(k+1) t^{-2}\right)}{2(\alpha(k+1))^{1-\frac{s}{2}}}, s<2,
$$

where $v(s, t)=\int_{0}^{t} x^{s-1} e^{-x} d x$ is the lower incomplete gamma function.

Further, the conditional moments, say $\Delta_{S}(t)$, is given by

$$
\Delta_{s}(t)=\int_{t}^{\infty} x^{s} f(x ; \varphi) d x
$$

Hence, by using pdf (3.3), we can write

$$
\Delta_{\mathrm{s}}(\mathrm{t})=\sum_{\mathrm{k}=0}^{\infty} w_{\mathrm{k}} \int_{\mathrm{t}}^{\infty} \mathrm{x}^{\mathrm{s}-3} \mathrm{e}^{-\alpha(\mathrm{k}+1) \mathrm{x}^{-2}} \mathrm{~d} x
$$

Then using the upper incomplete gamma function, we obtain

$$
\Delta_{\mathrm{s}}(\mathrm{t})=\sum_{\mathrm{k}=0}^{\infty} \mathfrak{w}_{\mathrm{k}} \frac{\Gamma\left(1-\frac{\mathrm{s}}{2}, \alpha(\mathrm{k}+1) \mathrm{t}^{-2}\right)}{2(\alpha(\mathrm{k}+1))^{1-\frac{s}{2}}}, \mathrm{~s}<2,
$$

where $\Gamma(s, t)=\int_{t}^{\infty} x^{s-1} e^{-x} d x$ is the upper incomplete gamma function.

\subsection{Inequality measures}

Lorenz and Bonferroni curves are the most widely used inequality measures in income and wealth distribution. In this subsection, we will calculated Lorenz, Bonferroni and Zenga curves for the OFIR distribution. The Lorenz, Bonferroni, and Zenga curves are obtained, respectively, as

$$
\begin{aligned}
& L_{F}(x)=\frac{\int_{0}^{t} x f(x) d x}{E(X)}=\frac{\sum_{k=0}^{\infty} w_{k} \frac{v\left(\frac{1}{2}, \alpha(k+1) t^{-2}\right)}{2(\alpha(k+1))^{\frac{1}{2}}}}{\sum_{k=0}^{\infty} \frac{w_{k} \sqrt{\pi}}{2[\alpha(k+1)]^{\frac{1}{2}}}}, \\
& B_{F}(x)=\frac{\int_{0}^{t} x f(x) d x}{E(X) F(x)}=\frac{L_{F}(x)}{F(x)}=\frac{\sum_{k=0}^{\infty} w_{k} \frac{\gamma\left(\frac{1}{2}, \alpha(k+1) t^{-2}\right)}{2(\alpha(k+1))^{\frac{1}{2}}}}{\left(\sum_{k=0}^{\infty} \frac{w_{k} \sqrt{\pi}}{2[\alpha(k+1)]^{\frac{1}{2}}}\right) e^{-\left(e^{\frac{\alpha}{x^{2}}}-1\right)^{\theta}}},
\end{aligned}
$$

and

$$
A_{F}(x)=1-\frac{\mu^{-}(x)}{\mu^{+}(x)}
$$

where

$$
\mu^{-}(x)=\frac{\int_{0}^{t} x f(x) d x}{E(X)}=\frac{\sum_{k=0}^{\infty} w_{k} \frac{v\left(\frac{1}{2}, \alpha(k+1) t^{-2}\right)}{2(\alpha(k+1))^{\frac{1}{2}}}}{\sum_{k=0}^{\infty} \frac{w_{k} \sqrt{\pi}}{2[\alpha(k+1)]^{\frac{1}{2}}}} \text { and } \mu^{+}(x)=\frac{\int_{t}^{\infty} x f(x) d x}{1-F(x)}=\frac{\sum_{k=0}^{\infty} w_{k} \frac{\Gamma\left(\frac{1}{2}, \alpha(k+1) t^{-2}\right)}{2(\alpha(k+1))^{\frac{1}{2}}}}{1-e^{-\left(e^{\frac{\alpha}{x^{2}}}-1\right)^{\theta}}} .
$$

\section{Maximum likelihood estimation}

The ML estimators of the unknown parameters for the OFIR distribution are determined based on complete samples. Let $X_{1}, \ldots, X_{n}$ be observed values from the OFIR distribution with set of parameters $\varphi=(\alpha, \theta)^{\top}$. The total log-likelihood function for the vector of parameters $\varphi$ can be expressed as 


$$
\ln L(\varphi)=n \ln 2 \theta+n \ln \alpha-3 \sum_{i=1}^{n} \ln x_{i}+\alpha \sum_{i=1}^{n} \frac{1}{x_{i}{ }^{2}}+(\theta-1) \sum_{i=1}^{n} \ln \left(e^{\frac{\alpha}{x_{i}{ }^{2}}}-1\right)-\sum_{i=1}^{n}\left(e^{\frac{\alpha}{x_{i}{ }^{2}}}-1\right)^{\theta} .
$$

The elements of the score function $\mathrm{U}(\varphi)=\left(\mathrm{U}_{\alpha}, \mathrm{U}_{\theta}\right)$ are given by

$$
\mathrm{u}_{\alpha}=\frac{n}{\alpha}+\sum_{i=1}^{n} \frac{1}{x_{i}{ }^{2}}+(\theta-1) \sum_{i=1}^{n} \frac{\frac{1}{x_{i}{ }^{2}} e^{\frac{\alpha}{x_{i}{ }^{2}}}}{e^{\frac{\alpha}{x_{i}{ }^{2}}}-1}-\theta \sum_{i=1}^{n} \frac{1}{x_{i}{ }^{2}} e^{\frac{\alpha}{x_{i}{ }^{2}}}\left(e^{\frac{\alpha}{x_{i}{ }^{2}}}-1\right)^{\theta-1},
$$

and

$$
\mathrm{u}_{\theta}=\frac{n}{\theta}+\sum_{i=1}^{n} \ln \left(e^{\frac{\alpha}{x_{i}^{2}}}-1\right)-\sum_{i=1}^{n}\left(e^{\frac{\alpha}{x_{i}^{2}}}-1\right)^{\theta} \ln \left(e^{\frac{\alpha}{x_{i}^{2}}}-1\right) .
$$

Then the ML estimators of the parameters $\alpha$ and $\theta$ are obtained by setting $U_{\alpha}$ and $U_{\theta}$ to be zero and solving them. Clearly, it is difficult to solve them, therefore applying the Newton-Raphson's iteration method and using the computer packages such as Maple or R or other softwares will solve them.

\section{Numerical results}

A numerical results is designed to evaluate and compare the behavior of the estimators with respect to their mean square errors (MSEs). We generate 3000 random sample $X_{1}, \ldots, X_{n}$ of sizes $n=(30,50,100,300)$ from OFIR distribution. Six choices sets of parameters are considered as: set 1:(0.5,0.5), set 2: $(1.5,0.5)$, set $3:(0.5,1.5)$, set $4:(1.5,1.5)$, set $5:(0.5,1)$, and set $6:(1,0.5)$.

The ML estimates of $\alpha$ and $\theta$ are computed. Then, the MSEs of the ML estimates (MLEs) of the unknown parameters are calculated. Simulated outcomes are listed in Table 1 and the following observations

\begin{tabular}{|c|c|c|c|c|c|c|c|}
\hline \multirow[b]{2}{*}{$n$} & \multirow{2}{*}{ Par } & \multicolumn{2}{|c|}{ set $1:(0.5,0.5)$} & \multicolumn{2}{|c|}{ set $2:(1.5,0.5)$} & \multicolumn{2}{|c|}{ set $3:(0.5,1.5)$} \\
\hline & & MLE & MSE & MLE & MSE & MLE & MSE \\
\hline \multirow{2}{*}{30} & $\bar{\alpha}$ & 0.5189 & 0.0095 & 0.6509 & 0.5736 & 1.5611 & 0.0828 \\
\hline & $\theta$ & 291 & 0.0184 & 1.5907 & 0.1757 & .5347 & 0.0211 \\
\hline \multirow{2}{*}{50} & $\alpha$ & 4 & 0.0060 & 5587 & 0.0766 & .5357 & 0.0520 \\
\hline & $\theta$ & 55 & 0.0104 & 1.5457 & 0.0954 & 0.5201 & 0.0109 \\
\hline \multirow{2}{*}{100} & $\alpha$ & 057 & 0.0031 & 0.5299 & 0.0196 & 1.5121 & 0.0253 \\
\hline & $\theta$ & 0.5091 & 0.0049 & 1.5303 & 0.0444 & 0.5076 & 0.0049 \\
\hline \multirow{2}{*}{300} & $\alpha$ & 0.5017 & 0.0010 & 0.5084 & 0.0048 & 1.5064 & 0.0089 \\
\hline & $\bar{\theta}$ & 0.5026 & 0.0015 & 1.5079 & 0.0140 & 0.5043 & 0.0016 \\
\hline \multirow[b]{2}{*}{$n$} & \multirow[b]{2}{*}{ Par } & \multicolumn{2}{|c|}{ set $4:(1.5,1.5)$} & \multicolumn{2}{|c|}{ set $5:(0.5,1)$} & \multicolumn{2}{|c|}{ set $6:(1,0.5)$} \\
\hline & & MLE & MSE & MLE & MSE & MLE & MSE \\
\hline \multirow{2}{*}{30} & $\alpha$ & 1.8977 & 5.6026 & 1.0350 & 0.0371 & 0.5518 & 0.0364 \\
\hline & $\theta$ & 1.5919 & 0.1705 & 0.5314 & 0.0199 & 1.0664 & 0.0829 \\
\hline \multirow{2}{*}{50} & $\alpha$ & 1.7085 & 0.6372 & 1.0214 & 0.0236 & 0.5287 & 0.0165 \\
\hline & $\bar{\theta}$ & 1.5500 & 0.0969 & 0.5159 & 0.0105 & 1.0388 & 0.0414 \\
\hline \multirow{2}{*}{100} & $\alpha$ & 1.5853 & 0.16 & 1.0106 & 0.0117 & 0.5119 & 0.0081 \\
\hline & $\theta$ & 1.5255 & 0.0428 & 0.5076 & 0.0049 & 1.0175 & 0.0203 \\
\hline \multirow{2}{*}{300} & $\alpha$ & 1.5219 & 0.0425 & 1.0039 & 0.0041 & 0.5058 & 0.0024 \\
\hline & $\bar{\theta}$ & 1.5073 & 0.0141 & 0.5038 & 0.0016 & 1.0069 & 0.0063 \\
\hline
\end{tabular}
are detected. The MSEs and the MLEs decrease as sample sizes increase for all estimates.

Table 1: The parameter estimation from OFIR distribution using MLmethod.

\section{Application}

In this section, we provide an application to a real data set to assess the flexibility of the OFIR model. In order to compare the OFIR model with other fitted distributions has four, five, and six parameters. 
We compare the fits of the OFIR distribution with the beta generalized inverse Weibull geometric distribution (BGIWGc) ( Elbatal et al., [7]), beta transmuted Weibull (BTW) (Afify et al., [3]), McDonald log-logistic (McLL) (Tahir et al., [18]), McDonald Weibull (McW) (Cordeiro et al., [6]), new modified Weibull (NMW) (Almalki and Yuan, [5]), transmuted complementary Weibull-geometric (TCWG) (Afify et al., [1]), beta Weibull (BW) (Lee et al., [17]), and exponentiated transmuted generalized Rayleigh (ETGR) (Afify et al., [2]) distributions.

The data set (Gross and Clark, [9]) on the relief times of twenty patients receiving an analgesic is 1.1, $1.4,1.3,1.7,1.9,1.8,1.6,2.2,1.7,2.7,4.1,1.8,1.5,1.2,1.4,3,1.7,2.3,1.6,2$.

The ML estimates along with their standard errors (SEs) of the model parameters are provided in Tables 2 and 3. In the same tables, the analytical measures including minus double log-likelihood $(-2 \log \mathrm{L})$, Anderson Darling statistic (A*), Cramér-von Mises statistic (W*), Akaike Information Criterion (AIC), corrected Akaike information criterion (CAIC), Bayesian information criterion (BIC), and Hannan-Quinn information criterion (HQIC) are presented.

Tables 2 lists the MLEs of the model parameters and their corresponding standard whereas errors the values of $-2 \log \mathrm{L}, \mathrm{AIC}, \mathrm{CAIC}, \mathrm{BIC}, \mathrm{HQIC}, \mathrm{A}^{*}$, and $\mathrm{W}^{*}$ are given in Table 3.

Table 2: MLEs and their SEs (in parentheses) for the data set.

\begin{tabular}{|l|c|c|c|c|c|c|}
\hline Model & \multicolumn{7}{|c|}{ MLE and SE } \\
\hline \multirow{2}{*}{ OFIR $(\boldsymbol{\alpha}, \boldsymbol{\theta})$} & 1.623 & 1.462 & - & - & - & - \\
& $(0.182)$ & $(0.265)$ & - & - & - & - \\
\hline \multirow{2}{*}{ BGIWGc $(\boldsymbol{\alpha}, \boldsymbol{\gamma}, \boldsymbol{\theta}, \mathrm{p}, \mathrm{a}, \mathrm{b})$} & 19.1874 & 20.5968 & 1.4346 & 9.8485 & $39.2308 \times 10^{-5}$ & 5.8015 \\
& $(33.03)$ & $(43.241)$ & $(0.837)$ & $(2.001)$ & $(63.252)$ & $(4.346)$ \\
\hline \multirow{2}{*}{ BTW $(\boldsymbol{\alpha}, \boldsymbol{\beta}, \mathrm{a}, \mathrm{b}, \boldsymbol{\lambda})$} & 5.6186 & 0.5311 & 53.3438 & 3.5683 & -0.7718 & - \\
& $(9.353)$ & $(0.148)$ & $(111.453)$ & $(4.265)$ & $(3.894)$ & - \\
\hline \multirow{2}{*}{ McLL $(\boldsymbol{\alpha}, \boldsymbol{\beta}, \mathrm{a}, \mathrm{b}, \mathrm{c})$} & 0.8811 & 2.0703 & 19.2254 & 32.0332 & 1.9263 & - \\
& $(0.109)$ & $(3.693)$ & $(22.341)$ & $(43.077)$ & $(5.165)$ & - \\
\hline \multirow{2}{*}{ McW $(\boldsymbol{\alpha}, \boldsymbol{\beta}, \mathrm{a}, \mathrm{b}, \mathrm{c})$} & 2.7738 & 0.3802 & 79.108 & 17.8976 & 3.0063 & - \\
& $(6.38)$ & $(0.188)$ & $(119.131)$ & $(39.511)$ & $(13968)$ & - \\
\hline \multirow{2}{*}{ NMW $(\boldsymbol{\alpha}, \boldsymbol{\beta}, \boldsymbol{\gamma}, \boldsymbol{\delta}, \boldsymbol{\theta})$} & 0.1215 & 2.7837 & $8.227 \times 10^{-5}$ & 0.0003 & 2.7871 & - \\
& $(0.056)$ & $(20.37)$ & $\left(1.512 \times 10^{-3}\right)$ & $(0.025)$ & $(0.428)$ & - \\
\hline \multirow{2}{*}{ TCWG $(\boldsymbol{\alpha}, \boldsymbol{\beta}, \boldsymbol{\gamma}, \boldsymbol{\lambda})$} & 43.6627 & 5.1271 & 0.2823 & -0.2713 & - & - \\
& $(45.459)$ & $(0.814)$ & $(0.042)$ & $(0.656)$ & - & - \\
\hline \multirow{2}{*}{ BW $(\boldsymbol{\alpha}, \boldsymbol{\beta}, \mathrm{a}, \mathrm{b})$} & 0.8314 & 0.6126 & 29.9468 & 11.6319 & - & - \\
& $(0.954)$ & $(0.34)$ & $(40.413)$ & $(21.9)$ & - & - \\
\hline \multirow{2}{*}{ ETGR $(\boldsymbol{\alpha}, \boldsymbol{\beta}, \boldsymbol{\lambda}, \boldsymbol{\delta})$} & 0.1033 & 0.6917 & -0.342 & 23.5392 & - & - \\
& $(0.436)$ & $(0.086)$ & $(1.971)$ & $(105.371)$ & - & - \\
\hline
\end{tabular}

Table 3: Measures of goodness-of-fit statistics for the data set.

\begin{tabular}{|l|c|c|c|c|c|c|c|}
\hline Model & $-2 \log$ L & AIC & CAIC & BIC & HQIC & $A^{*}$ & $W^{*}$ \\
\hline OFIR & 31.476 & 35.476 & 36.181 & 34.078 & 35.864 & 0.23635 & 0.0399 \\
\hline BGIWGc & 31.662 & 43.662 & 50.124 & 39.468 & 44.828 & 0.24665 & 0.0434 \\
\hline BTW & 33.051 & 43.051 & 47.337 & 39.556 & 44.023 & 0.39769 & 0.06896 \\
\hline McLL & 33.854 & 43.854 & 48.14 & 40.359 & 44.826 & 0.46199 & 0.07904 \\
\hline McW & 33.907 & 43.907 & 48.193 & 40.412 & 44.879 & 0.46927 & 0.08021 \\
\hline NMW & 41.173 & 51.173 & 55.459 & 47.678 & 52.145 & 1.0678 & 0.17585 \\
\hline TCWG & 33.607 & 41.607 & 44.274 & 38.811 & 42.385 & 0.43603 & 0.07252 \\
\hline BW & 34.396 & 42.396 & 45.063 & 39.6 & 43.174 & 0.51316 & 0.0873 \\
\hline ETGR & 36.856 & 44.856 & 47.523 & 42.06 & 45.634 & 0.79291 & 0.13629 \\
\hline
\end{tabular}



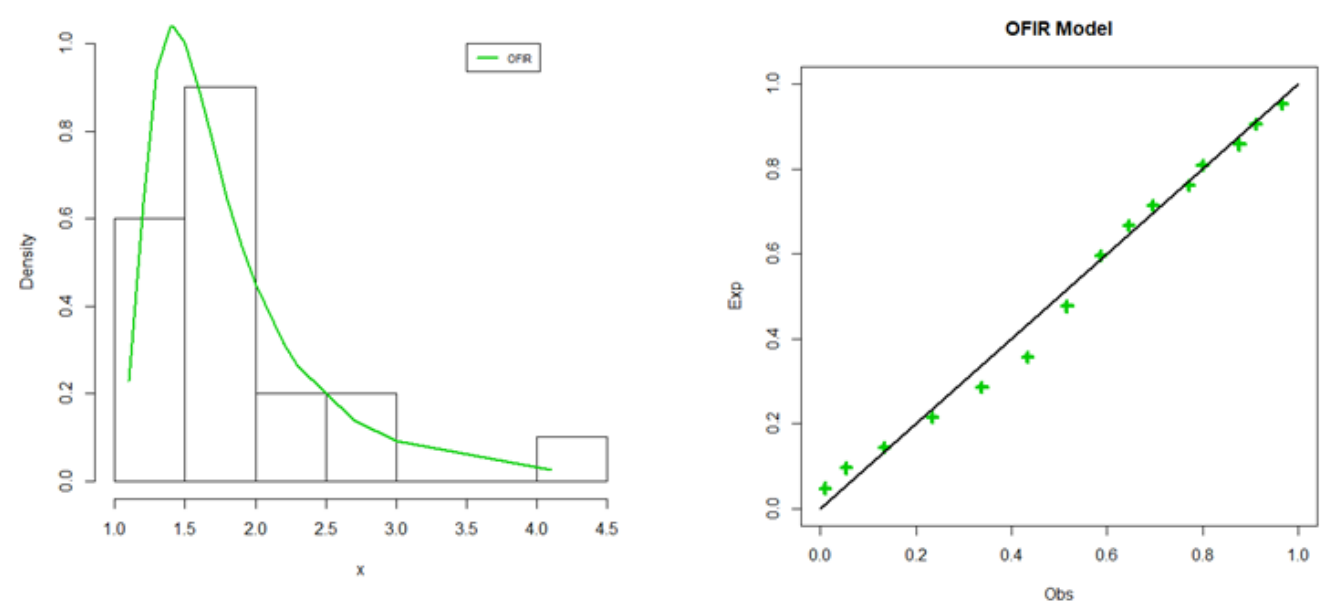

Figure 2: The empirical pdf and pp plots of the OFIR model.
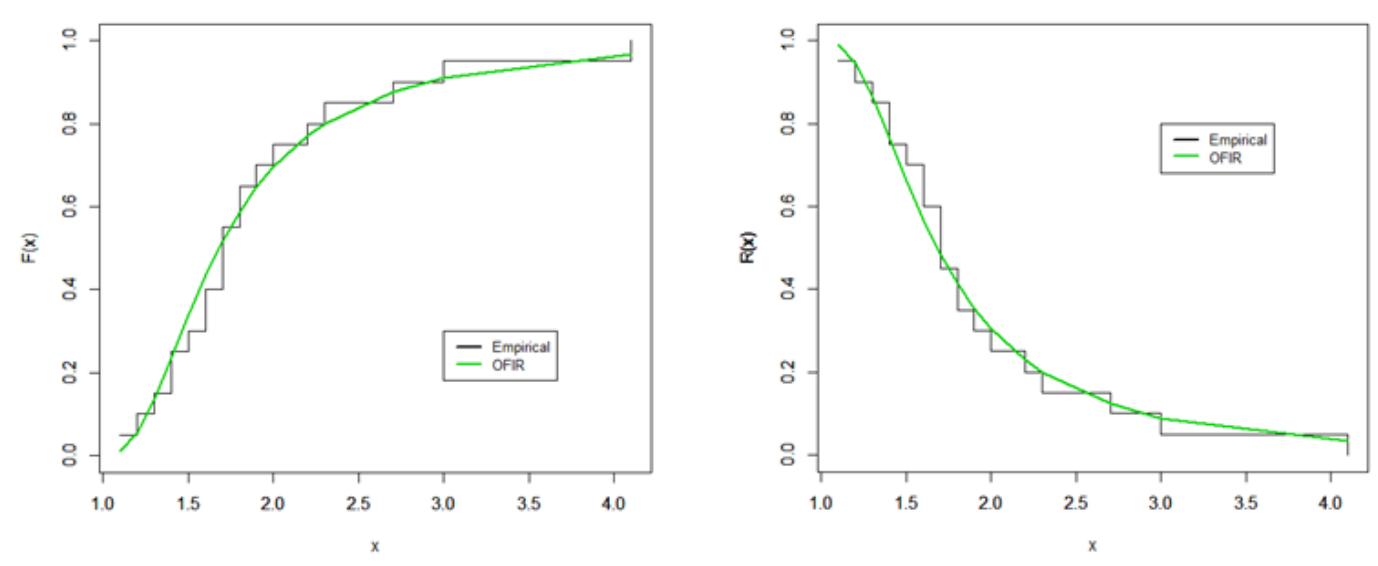

Figure 3: The empirical cdf and sf of the OFIR model.

Table 3 compares the fits of the OFIR distribution with the BGIWGc, BTW, McLL, McW, NMW, TCWG, BW and ETGR distributions. The figures in these tables show that the OFIR model has the lowest values for $-2 \log$ L, AIC, CAIC, HQIC, $A^{*}$, and $W^{*}$ among all fitted distributions. So, it could be chosen as the best model. The fitted pdf and pp plots for the OFIR model are displayed in Figure 2. Figure 3 shows the estimated cdf and sf for the OFIR model. From these plots it is evident that the new model provides close fit to the data.

\section{Concluding Remarks}

In this paper, we propose a new two-parameter distribution named the odd Fréchet inverse Rayleigh distribution. The pdf of OFIR can be expressed as a linear mixture of IR densities. We calculate explicit expressions for some of its statistical properties. We study the maximum likelihood estimation. Simulation results are carried to assess the accuracy and performance of estimates. The proposed model provides better fits than some other competitive models using a real data set. We wish that the proposed distribution would attract wider applications in applied areas such as lifetime analysis, reliability, hydrology, and engineering. 


\section{References}

[1] A. Z. Afify, Z. M. Nofal, N. S. Butt, Transmuted complementary Weibull geometric distribution, Pak. J. Stat. Oper. Res., $10(2014), 435-454.6$

[2] A. Z. Afify, Z. M. Nofal, A. N. Ebraheim, Exponentiated transmuted generalized Rayleigh distribution: A new four parameter Rayleigh distribution, Pak. J. Stat. Oper. Res., 11 (2015), 115-134. 6

[3] A. Z. Afify, H. M. Yousof, S. Nadarajah, The beta transmuted-H family for lifetime data, Stat. Interface, 10 (2017), 505-520. 6

[4] A. Ahmad, S. P. Ahmad, A. Ahmed, Transmuted inverse Rayleigh distribution: A generalization of the inverse Rayleigh distribution, Math. Theo. Model., 4 (2014), 90-98. 1

[5] S. J. Almalki, J. Yuan, A new modified Weibull distribution, Reliab. Eng. Syst. Safety, 111 (2013), 164-170. 6

[6] G. M. Cordeiro, E. M. Hashimoto, E. M. M. Ortega, The McDonald Weibull model, Statistics, 48 (2014), 256-278. 6

[7] I. Elbatal, Y. M. El Gebaly, E. A. Amin, The beta generalized inverse Weibull geometric distribution and its applications, Pak. J. Stat. Oper. Res., 13 (2017), 75-90. 6

[8] M. K. Gharraph, Comparison of estimators of location measures of an inverse Rayleigh distribution, Egyptian Statist. J., 37 (1993), 295-309. 1

[9] A. J. Gross, V. A. Clark, Survival distributions: Reliability applications in the biomedical sciences, John Wiley \& Sons, New York, (1975). 6

[10] M. A. Haq, Kumaraswamy exponentiated inverse Rayleigh distribution, Math. Theo. Model., 6 (2016), 93-104. 1

[11] M. A. Haq, Transmuted exponentiated inverse Rayleigh distribution, J. Stat. Appl. Prob., 5 (2016), 337-343. 1

[12] M. A. Haq, M. Elgarhy, The odd Fréchet-G family of probability distributions, J. Stat. Appl. Prob., 7 (2018), 185-201. 1, 3.2

[13] A. S. Hassan, E. A. Amin, A. A. Abd-El Aziz, Estimation and prediction from inverse Rayleigh distribution based on lower record values, Appl. Math. Sci. (Ruse), 4 (2010), 3057-3066. 1

[14] M. S. Khan, Modified inverse Rayleigh distribution, Int. J. Comput. Appl., 87 (2014), 28-33. 1

[15] M. S. Khan, R. King, Transmuted modified inverse Rayleigh distribution, Austrian J. Statist., 44 (2015), 17-29. 1

[16] J. Leao, H. Saulo, M. Bourguignon, R. Cintra, L. C. Rego, G. M. Cordeiro, On some properties of the beta inverse Rayleigh distribution, Chil. J. Stat., 4 (2013), 111-131. 1

[17] C. Lee, F. Famoye, O. Olumolade, Beta-Weibull distribution: Some properties and applications to censored data, J. Modern Appl. Statist. Methods, 6 (2007), 173-186. 6

[18] M. H. Tahir, M. Mansoor, M. Zubair, G. G. Hamedani, McDonald log logistic distribution with an application to breast cancer data, J. Stat. Theory Appl., 13 (2014), 65-82. 6

[19] V. N. Trayer, Doklady Acad, Nauk, Belorus, U. S. S. R. (1964). 1

[20] V. G. Voda, On the inverse Rayleigh distributed random variable, Rep. Statist. Appl. Res. Un. Japan. Sci. Engrs., 19 (1972), 13-21. 1 